\title{
Confronting the Challenge of Integrated Assessment of Climate Adaptation: A Conceptual Framework
}

\author{
Ian Sue Wing \\ Boston University \\ Karen Fisher-Vanden \\ Pennsylvania State University
}

\begin{abstract}
Key limitations of integrated assessment models (IAMs) are their highly stylized and aggregated representation of climate damages and associated economic responses, as well as the omission of specific investments related to climate change adaptation. This paper proposes a framework for modeling climate impacts and adaptation that clarifies the relevant research issues and provides a template for making improvements. We identify five desirable characteristics of an ideal integrated assessment modeling platform, which we elaborate into a conceptual model that distinguishes three different classes of adaptation-related activities. Based on these elements we specify an impacts- and adaptation-centric IAM, whose optimality conditions are used to highlight the types of functional relationships necessary for realistic representations of adaptation-related decisions, the specific mechanisms by which these responses can be incorporated into IAMs, and the ways in which the inclusion of adaptation is likely to affect the simulations' results.
\end{abstract}




\section{Introduction}

Integrated assessments of climate change have concentrated on developing baseline scenarios of future greenhouse gas (GHG) emissions, and analyzing the economic consequences of emission mitigation policies. A pervasive limitation of existing integrated assessment models (IAMs) is their highly stylized and aggregated representation of climate impacts and the economic responses thereto, and the omission of specific investments related to climate change adaptation. This state of affairs originates in our incomplete understanding of the channels through which anthropogenic radiative forcing induces changes in meteorological variablesand through these, various other biophysical impact endpoints at regional scales_-as well as what the concomitant damages to the various economic sectors within these regions might be. The good news is that this situation is slowly improving, with several advances made over the past decade to introduce impacts and adaptation into IAMs. However, systemic challenges to modeling adaptation continue to impede progress in this area.

In this paper we propose a conceptual framework for modeling climate impacts and adaptation. Our goal is to clarify the relevant research issues and provide a useful template for making progress in this difficult but vital area. To this end we address three important questions: what types of functional relationships do IAMs need to include in order to more realistically represent the kinds of adaptation-related decisions that need to be made, what are specific mechanisms by which these responses can be incorporated into models, and how and why is their inclusion likely to affect IAM results?

The rest of the paper is organized as follows. Section 2 provides the motivation for our inquiry, discussing the special challenges that attend modeling the processes of adapting to climate change. In section 3 we introduce our conceptual framework for understanding and 
analyzing climate impacts and adaptation. In section 4 we elaborate the core elements of our framework into a stylized IAM whose optimality conditions highlight the channels through which climate impacts' and adaptation exert effects on the economy. Section 5 expands on these results, offering additional observations and discussion. Section 6 concludes with a brief summary.

\section{Confronting the Challenges of Modeling Adaptation}

What are the unique features of climate adaptation, and what special challenges do they pose to integrated assessment modeling? The fundamental premise of this paper is that the biophysical impacts of climate change will be heterogeneous, both in terms of their attributes and their geographic incidence, resulting in shocks to natural and human systems that differ in character and magnitude across regions. ${ }^{1}$ Climate damages are also likely to be sector-specific, with particular categories of economic activity (e.g., agriculture, coastal settlements) being more severely impacted than others. Furthermore, because adaptation will likely target defensive expenditures to particular damage endpoints in regions and sectors anticipated to be particularly exposed and vulnerable, such investments are likely to be regionally and sectorally differentiated as well. To capture such decisions, an ideal IAM would need to contain sufficient regional and sectoral detail to resolve the variation in the relevant climate impacts and responses, or at least be able to consistently aggregate these artifacts up to coarser spatial scales.

A second issue is the character of adaptational responses to these impacts. Adaptation comes in different forms: passive general market reactions (e.g., changing heating and cooling expenditures or shifts in choice of tourism destinations); specific reactive adaptation investments (e.g., treatment of vector-borne disease); and specific proactive adaptation investments (e.g., the

\footnotetext{
${ }^{1}$ E.g., areas that are currently near the boundaries of different climate regimes will likely experience the largest climatic changes, and impacts, as these regimes shift.
} 
hardening of vulnerable infrastructure, development of early warning systems, and expansion of capacity for climate-related disaster preparedness and response). A few IAMs already embody the capability to simulate passive adaptation through endogenous market responses to climateinduced changes in prices. Examples include increases in electricity generation in response to higher residential and commercial cooling demands triggered by summer heat waves, or decline in rain-fed crop yields induced by shocks to the productivity of agricultural land from reduced precipitation. To simulate adaptation existing IAMs need to incorporate a region-by-sector array of climate impacts each element of which represents the relevant shock to the economy.

Thirdly, endogenous investment in proactive adaptation measures such as coastal protection is typically absent in IAMs due to difficulties modeling intertemporal decision making under uncertainty. Proactive adaptation is influenced by economic actors' expectations of the timing and magnitude of future climate damages. Unlike reactive adaptation, these measures are investments designed to protect against future impacts that are far from certain, and therefore generate protection that is cumulative and inherently intertemporal in nature. To approximate investment trajectories that are optimal given the current information set, IAMs should be capable of capturing the tradeoff between the discounted benefits of avoided future damages and the opportunity cost of investments today. Furthermore, because investment decisions depend on both expectations of impacts and the rate of time preference, they are inextricably linked to decisions to emit or abate greenhouse gases (GHGs) in the near term.

Fourth, increases in the demand for adaptation will likely induce technological improvements in adaptation-related activities. How much this kind of innovation differs significantly from mitigation-related technological change is not well understood. A particular question is whether the regional and sectoral specificity of adaptation measures, coupled with the 
risk to public infrastructure from climate-related damages, might end up limiting the market for new adaptation techniques, reducing the attractiveness of private $R \& D$ and increasing the role for public investments. The public-private distinction is more than a question of simply basic versus applied science — as with government support for defense-related R\&D, adaptation-related innovations will be driven by the nature of demand for the final product. IAMs should be able to represent relevant differences on the demand side, as well as the revenue-raising mechanisms that supply public investment funds.

Finally, IAM representations of climate impacts' economic effects and the adaptation's mitigating influence should ideally be grounded in empirical evidence. Although climate impacts and adaptation is the subject of a burgeoning empirical literature, this work is mostly restricted to a few regions and sectors, and tends to focus on the direct impacts of temperature on economic outcomes, with limited ability to disentangle the contributions of specific biophysical endpoints. The upshot is a disconnect between empirical estimates and IAMs, with attendant difficulties in translating the former into functional forms and parameters useful to the latter. Furthermore, there are only a few empirical assessments of adaptation-related technological improvements that have been widely recognized to have significantly lowered the cost of responding to—or defending against—climate damages. ${ }^{2}$ This deficit reflects our limited understanding of the process by which technologies of this kind improve, especially if innovations may be targeted toward specific impact endpoints. Thus, in empirical research there is a need for not only broader regional and sectoral coverage, but also deeper insight into the specific channels through which changes in climate variables generate impacts on the economy.

\footnotetext{
${ }^{2}$ E.g., Landon-Lane et al (2011) conclude that banking system innovations post-1940 reduced the correlation between climate impacts on agricultural production and financial distress in the U.S. Midwest. Fishback et al (2011) find access to information to be a significant attenuator of temperature's influence on mortality, providing support for the beneficial effect of past U.S. public health campaigns. Hansen et al (2011) find that irrigation and dam infrastructure mitigated the impacts of precipitation extremes on agricultural yields.
} 


\section{Climate Impacts and Adaptation: A Bottom-Up Conceptual Model}

A simple conceptual framework that encompasses the foregoing points is illustrated in Figure 1. Starting from the top, human induced increases in atmospheric GHG concentrations (i) drive changes in climate variables such as temperature and precipitation at the regional scale (ii). These climatic changes result in physical impacts (iii) which influence the productivity of various sectors of the regional economies where the impacts occur (iv), generating economic losses (v).

Less straightforward is how adaptation activities induced by the threat, or the onset, of economic damages arise endogenously. The response of sectoral productivities to the character and magnitude of the initiating physical impacts is moderated by specific protective or defensive measures (e.g., coastal protection infrastructure to defend against rising seas, or the development of drought- and heat-tolerant varieties of staple crops), which we refer to as Type II adaptation activities. A qualitatively different type of adaptation lessens the adverse effects of the impacts that do occur on economic sectors' productivity. We refer to specific investments of this kind as Type III measures, which include insurance, redundant or flexible production capacity, or investments in disaster preparedness, response and recovery. Lastly, for given levels of these specific adaptations (or no adaptation), the magnitude of the damages that do ultimately befall the economy also depend upon price changes and substitution responses across many markets. These passive general equilibrium adjustments should be considered as a distinct form of adaptation, which we label Type I. Crucially, the three types of responses interact with one another, and are not separable from the overarching context of the magnitude and character of the climate impacts that generate the demand for adaptation in the first place. This suggests that to quantify the economic consequences of climate impacts we must first characterize how key 
impact endpoints depend on changes in climate variables at the regional scale (B) and how sectoral productivity shocks depend on these endpoints (C).

To formally model this intuition, we use subscripts $m, j, \ell, i$ and $t$ to index the sets of climate-related variables, economic sectors, geographic locations, impact endpoints, and time periods. We can think of (B) as a family of mathematical response functions $\left(\zeta_{j, \ell}^{i}\right)$ which translate climate variables $\left(M_{\ell, t}^{m}\right)$ into biophysical impact endpoints $\left(b_{j, \ell, t}^{i}\right)$. Likewise, (C) is a family of functions, $\lambda_{j, \ell}^{i}$, that translate impacts into shocks to productivity $\left(\Lambda_{j, \ell, t}\right)$. The functions $\zeta_{j, \ell}^{i}$ and $\lambda_{j, \ell}^{i}$ are response surfaces in the form of Table 1 whose full exploitation requires a multisectoral representation of economic activity—for example, production functions denominated by region and sector $\left(\psi_{j, \ell}\right)$. $^{3}$

In this context it is tempting to interpret Type II “shielding” investments as proactive stock adaptation and Type III “coping” adaptations as reactive flow adaptation, but this would not be strictly correct. Each category will in general include a mix of proactive and reactive measures, with the essential difference being the mechanism through which each type exerts its moderating effect. Protective measures lessen sectors' exposure by attenuating the marginal effects of impact endpoints on productivity changes $\left(\partial \zeta_{j, \ell}^{i} / \partial M_{\ell, t}^{m}\right)$; coping measures increase resilience by attenuating the marginal effects of productivity shocks on economic losses $\left(\partial \lambda_{j, \ell}\right)$ $\left.\partial b_{j, \ell, t}^{i}\right)$.

This distinction has potentially important implications for the allocation of adaptation investments under uncertainty. Planning to limit a sector's exposure to climate damage requires an understanding of how specific impact endpoints affect its productivity. There may also be

\footnotetext{
${ }^{3}$ The state of the art in this regard is the FUND model's "impact sectors" (Tol, 1995; Anthoff et al, 2011), but this approach tends to conflate biophysical endpoints with the parts of the economy they might affect. The concern is that some impacts might induce substitution of output from more-exposed to less-exposed industries.
} 
other "general purpose" investments that are not climate-specific but lower the cost of adjustment to many different kinds of shocks. If adaptive investments prove to be more generic and fungible, while defensive investments are more impact-, sector- and region-specific, then we would expect more of the former and less of the latter. But conjectures such as these await empirical confirmation. A potentially fruitful direction for future research is to characterize the distribution of historical expenditures on adaptation to natural hazards, climatic and otherwise (see, e.g., Libecap and Steckel, 2011).

\section{Implementation: A Canonical IAM}

The potential for regional and sectoral heterogeneity in climate impacts and adaptation suggests that a single global or economy-wide damage function of the kind employed by many IAMs is insufficient. In this section we illustrate an alternative specification of impacts and damages in the context of a stylized IAM. For clarity and simplicity, we focus on impacts and adaptation responses related to sectoral productivity, and do not seek to explicitly represent all of the elements of Figure 1 in the fullest possible detail. Instead, where appropriate, we illustrate opportunities to adapt the model to explicitly highlight impacts and adaptation responses affecting factor inputs and market exchange.

Figure 2 presents our canonical IAM, which extends the well-known RICE and ADWITCH models (Nordhaus, 2010; Bosello et al, 2010a,b). The outputs of regional economies expand due to technical progress, capital accumulation and the extraction of geological carbonenergy resources, driving accumulation of a stock of atmospheric GHGs that generate climate damages. The main feedback is the influence of current global fossil energy use on the regional and sectoral distribution of future productivities via the climate system (1j)-(1k), the biophysical impacts of climate change (1l) and consequent shocks to the economy (1m). There are two novel 
features: a one-to-one elaboration of endpoint-sector damage linkages through the sectoral production functions (1c), as well as expenditures on contemporaneous reactive and forwardlooking proactive adaptation $\left(\rho_{j, \ell, t}^{i}\right.$ and $\left.\pi_{j, \ell, t}^{i}\right)$, the second of which accumulates into a stock of adaptation capital $\left(a_{j, \ell, t}^{i}\right)$. As discussed above, stock and flow adaptation can be both protective and ameliorative, via their moderating effects on impacts (1l) and subsequent damages (1m).

Our model specification is not without sacrifices. We omit labor as an explicit input to production, primarily to reduce notational clutter, but also because most IAMs treat the growth of population and the supply of labor in natural units as exogenous. ${ }^{4}$ Similarly, we subsume secular trends in aggregate productivity or energy-efficiency improvement implicitly within our sectoral production functions in order to focus narrowly on the effects of climate damages. We model the latter as adverse productivity shocks that have the potential to influence both the rate and bias of sectors' technical progress, and, over time the sectoral composition of the economy. ${ }^{5}$ This deliberate simplification does not provide an explicit representation of either demand-side impacts such as increases in the use of electricity for air conditioning in response to higher temperatures or capital stock destruction due to extreme events such as tropical cyclones. ${ }^{6}$ The validity of this modeling strategy turns on the degree of regional disaggregation—while it is difficult to imagine extreme events that are so destructive as to appreciably affect aggregate capital accumulation in large regions such as the US or China, biases may arise in models with sub-national detail.

\footnotetext{
${ }^{4}$ Including labor would create the equivalent of an additional "fixed factor" in eq. (1c), which would not generate additional insight. We note that our existing formulation is nonetheless able to accommodate in an implicit fashion the potential output effects of climate impacts on labor productivity (e.g., Graff Zivin and Neidell, 2010).

${ }^{5}$ Compare the special case of DICE/RICE-type IAMs in which damages have a neutral effect, which in the current notation can be specified as $q_{j, l, t}^{Y}=\Lambda_{j, l, t} \cdot \psi_{j, l}\left[q_{j, l, t}^{E}, q_{j, l, t}^{K}\right]$.

${ }^{6}$ These could potentially be captured through modifications to the sectoral output aggregation functions (1b) or the perpetual inventory equations (1h).
} 
The primary economic control variables are aggregate physical capital investment $\left(X_{\ell, t}^{K}\right)$ and carbon-energy resource extraction $\left(Q_{\ell, t}^{E}\right)$, while the primary environmental controls are investment in proactive and reactive adaptation. (There is also the intersectoral allocation of capital and energy $-q_{j, \ell, t}^{K}$ and $q_{j, \ell, t}^{E}$, but this is of secondary importance, and not discussed further.) We go on to derive the optimality conditions associated with the primary controls, and, inspired by Golosov et al (1011) and Hassler and Krusell (1012), use the results to understand the drivers of climate damages and adaptation's moderating role. Throughout, we focus on a reference sector $j^{\prime}$, region $\ell^{\prime}$ and time period $t^{\prime} .7$

\section{Physical capital investment}

Capital accumulation is the primary engine of economic growth apart from secular productivity increase. The condition for optimal investment is $\partial \mathcal{W} / \partial X_{\ell^{\prime}, t^{\prime}}^{K}=0$, which yields:

$$
\begin{aligned}
& \beta^{t^{\prime}} \frac{\partial \Xi}{\partial U_{\ell^{\prime}}} \cdot \frac{\partial U_{\ell^{\prime}}}{\partial Q_{\ell^{\prime}, t^{\prime}}^{C}} \\
& \quad=\mathbb{E}_{t^{\prime}} \sum_{t=t^{\prime}+1}^{T} \beta^{t}\left(1-\vartheta^{K}\right)^{t-t^{\prime}-1} \frac{\partial \Xi}{\partial U_{\ell^{\prime}}} \cdot \frac{\partial U_{\ell^{\prime}}}{\partial Q_{\ell^{\prime}, t}^{C}} \cdot \sum_{j=1}^{\mathcal{N}}\left(\frac{\partial \Phi_{\ell^{\prime}}}{\partial q_{j, \ell^{\prime}, t}^{Y}} \cdot \frac{\partial \psi_{j, \ell^{\prime}, t}}{\partial q_{j, \ell^{\prime}, t}^{K}} \cdot \frac{\partial q_{j, \ell^{\prime}, t}^{K}}{Q_{\ell^{\prime}, t}^{K}}\right)
\end{aligned}
$$

\footnotetext{
${ }^{7}$ Eq. (1j) can be used to substitute for $G$ in (1k), and the outcome can be plugged into (1l) for $M^{m}$. The new eq. (1l) can be plugged into $(1 \mathrm{~m})$ to eliminate $b^{i}$. The result, which expresses regional and sectoral climate shocks as a function of lagged values of carbon-energy, as well as current investment in reactive adaptation and the extant stock of proactive adaptation capacity, can be used to eliminate $\Lambda$ in (1c). Eq. (1c) can be further reduced by using (1d) and (1e) to express inputs of carbon-energy and capital to sectoral production as implicit functions of their aggregate supplies, $Q^{K}$ and $Q^{E}$. And (1h) and (1i) can be used to express the current state variables $Q^{K}$ and $\boldsymbol{a}$ as functions of the lagged values of their respective controls, $X^{K}$ and $\pi$. The new eq. (1c) can be used to substitute for $q^{Y}$ in (1b) and the result plugged into (1f) to eliminate $Q^{Y}$. Finally, (1g) can be substituted into (1f) to eliminate $P^{E}$. The new eq. (1f) can then be used to substitute for $Q^{C}$ in (1a), allowing our IAM to be approximated as an unconstrained maximization problem in which the objective is denominated over the four control variables:
}

$$
\mathcal{W}=\mathbb{E}_{0} \sum_{t=0}^{T} \beta^{t} \Xi\left[\ldots, U_{\ell^{\prime}}\left[X_{\ell^{\prime}, t-1}^{K}, X_{\ell^{\prime}, t-2}^{K}, \ldots, Q_{\ell^{\prime}, t^{\prime}}^{E}, Q_{\ell^{\prime}, t-1}^{E}, \ldots, \boldsymbol{\rho}_{j, \ell^{\prime}, t}, \boldsymbol{\pi}_{j, \ell^{\prime}, t-1}, \boldsymbol{\pi}_{j, \ell^{\prime}, t-2}, \ldots\right], \ldots\right] .
$$


On the left is the period- $t^{\prime}$ marginal cost of investment (forgone current consumption), while on the right is the present value of the output increases generated by the increment to the capital stock. Through the sectoral production functions (1c), climate-induced adverse shocks depress the marginal product of capital $\left(\partial \psi_{j, \ell^{\prime}, t} / \partial q_{j, \ell^{\prime}, t}^{K}\right)$, attenuating aggregate near-term investment by reducing its beneficial effect on future output. Conversely, the ability to adapt limits the expected decline in capital's marginal product, thereby increasing investment.

We draw additional insights by calculating the optimal demand for contemporaneous investment. Key to our method is the elasticities summarized in Table 2. Let $\gamma_{1}-\gamma_{5}$ represent the instantaneous elasticities of $\ell^{\prime}$ 's aggregate output with respect to the output of sector $j^{\prime}$, sectoral output with respect to its own use of capital, $j^{\prime \prime}$ s use of capital with respect to the aggregate capital supply (capturing the effects of reallocation), and the investment elasticity of future capital supply. ${ }^{8}$ Finally, define the region $\ell^{\prime}$ aggregate price deflator, $\mathcal{D}_{\ell^{\prime}, t, t^{\prime}}=\frac{\partial U_{\ell^{\prime}}}{\partial Q_{\ell^{\prime}, t}^{C}} / \frac{\partial U_{\ell^{\prime}}}{\partial Q_{\ell^{\prime}, t^{\prime}}^{C}}$ as the index of change in the marginal utility of consumption, and $g_{\ell^{\prime}, t, t^{\prime}}^{Y}=Q_{\ell^{\prime}, t}^{Y} / Q_{\ell^{\prime}, t^{\prime}}^{Y}$ as its future GDP growth. Rearranging (3) yields the optimal investment intensity of GDP:

$$
\begin{aligned}
\frac{X_{\ell^{\prime}, t^{\prime}}^{K}}{Q_{\ell^{\prime}, t^{\prime}}^{Y}}=\mathbb{E}_{t^{\prime}} & \sum_{t=t^{\prime}+1}^{T} \beta^{t-t^{\prime}} \mathcal{D}_{\ell^{\prime}, t, t^{\prime}} g_{\ell^{\prime}, t, t^{\prime}}^{Y} \gamma_{4}\left[Q_{\ell^{\prime}, t}^{K}, X_{\ell^{\prime}, t^{\prime}}^{K}\right] \\
& \times \sum_{j=1}^{\mathcal{N}}\left(\gamma_{1}\left[Q_{\ell^{\prime}}^{Y}, q_{j, \ell^{\prime}}^{Y}\right] \cdot \gamma_{2}\left[q_{j, \ell^{\prime}}^{Y}, q_{j, \ell^{\prime}}^{K}\right] \cdot \gamma_{3}\left[q_{j, \ell^{\prime}}^{K}, Q_{\ell^{\prime}}^{K}\right]\right)
\end{aligned}
$$

The second summation on the right-hand side is the elasticity of future aggregate output with respect to aggregate capital, mediated via the sectoral structure of the economy. The larger the value of this variable, or future output growth, the greater the intensity of physical capital

\footnotetext{
${ }^{8}$ The marginal effect of investment at $t^{\prime}$ on subsequent capital stocks is $\left(1-\vartheta^{K}\right)^{t-t^{\prime}-1}=\gamma_{4}\left[Q_{\ell, t}^{K}, X_{\ell, t^{\prime}}^{K}\right]$. $Q_{\ell, t}^{K} / X_{\ell, t^{\prime}}^{K}$
} 
investment. The same is true for the discount factor, the future aggregate price level and the durability of current investment. We take pains to emphasize that the elasticities in this expression should not be thought of as numerical constants. Rather, their instantaneous values are functions of their second argument and the modulating influences of intervening variables. A case in point is the capital-output elasticity $\left(\gamma_{2}\right)$, whose values depends on the impact on the benchmark capital accumulation path of the carbon-energy extraction rate and the productivity impacts of climate damages and adaptation. ${ }^{9}$ The Type I adaptations thus induced will be the changes in the path of investment and the intersectoral allocation of energy and capital.

\section{Carbon-energy extraction}

RICE and DICE specify a Leontief relationship between aggregate output and inputs of carbon-energy, the coefficient on which declines at an exogenously-specified rate of energy efficiency improvement. The resulting progressive decoupling of emissions from output is the central moderating force on atmospheric GHG accumulation and future climate damages. In the present model carbon-energy extraction is a decision variable influenced by the endogenous marginal productivity of energy use. Optimal extraction is given by $\partial \mathcal{W} / \partial Q_{\ell^{\prime}, t^{\prime}}^{E}=0$, yielding:

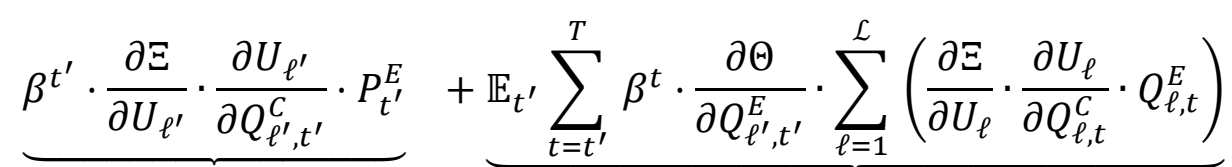

(a) Contemporaneous marginal extraction cost
(b) Resource stock effect of contemporaneous energy use

\footnotetext{
${ }^{9}$ Obviously, there is also uncertainty associated with the distribution of future output growth across sectors and regions $\left(g^{Y}\right)$, but this is comparatively straightforward to address through Monte Carlo simulation, even in existing IAMs.
} 


$$
-\mathbb{E}_{t^{\prime}} \sum_{t=t^{\prime}+1}^{T} \beta^{t} \sum_{\ell=1}^{\mathcal{L}}\left\{\frac{\partial \Xi}{\partial U_{\ell}} \cdot \frac{\partial U_{\ell}}{\partial Q_{\ell, t}^{C}} \cdot \sum_{j=1}^{\mathcal{N}}\left\{\times \sum_{i=1}^{J}\left[\begin{array}{c}
\frac{\partial \Phi_{\ell}}{\partial q_{j, \ell, t}^{Y}} \cdot \frac{\partial \psi_{j, \ell, t}}{\partial \Lambda_{j, \ell, t}} \\
\frac{\partial \lambda_{j, \ell}}{\partial b_{j, \ell, t}^{i}} \\
\left.\left.\times \sum_{m=1}^{\mathcal{M}}\left(\frac{\partial \zeta_{j, \ell}^{i}}{\partial M_{\ell, t}^{m}} \cdot \sum_{s=t^{\prime}+1}^{t} \frac{\partial Y_{\ell, t}^{m}}{\partial G_{s}} \cdot \frac{\partial \varepsilon_{s}}{\partial Q_{\ell^{\prime}, t^{\prime}}^{E}}\right)\right]\right\}
\end{array}\right\}\right\}\right.
$$

(c) Present value of future marginal damages from climate change

$$
=\underbrace{\beta^{t^{\prime}} \cdot \frac{\partial \Xi}{\partial U_{\ell^{\prime}}} \cdot \frac{\partial U_{\ell^{\prime}}}{\partial Q_{\ell^{\prime}, t^{\prime}}^{C}} \cdot \sum_{j=1}^{\mathcal{N}}\left(\frac{\partial \Phi_{\ell^{\prime}}}{\partial q_{j, \ell^{\prime}, t^{\prime}}^{Y}} \cdot \frac{\partial \psi_{j, \ell^{\prime}}}{\partial q_{j, \ell^{\prime}, t^{\prime}}^{E}} \cdot \frac{\partial q_{j, \ell^{\prime}, t^{\prime}}^{E}}{\partial Q_{\ell^{\prime}, t^{\prime}}^{E}}\right)}
$$

Contemporaneous marginal benefit

On the right is the contemporaneous marginal benefit, while the left is the marginal social cost of carbon-energy, made up of (a) the marginal extraction cost, (b) the stock effect of current energy use on the future marginal extraction costs, and (c) the marginal external cost of climate damage from carbon-energy consumption. The last component is the "social cost of carbon" (SCC), which, because it emanates from a globally well-mixed pollutant, does not depend on where energy is extracted or consumed. The sign of (c) reflects the generally negative effect of climate shocks on production $\left(\partial \psi_{j, \ell, t} / \partial \Lambda_{j, \ell, t} \leq 0\right)$.

We go on to derive the contemporaneous value of carbon-energy extraction as a share of GDP. Turning again to Table $2, \gamma_{5}$ and $\gamma_{6}$ represent the contemporaneous elasticities of sectoral output with respect to energy use and sectoral with respect to aggregate energy use, and $\gamma_{8}-\gamma_{10}$ represent the contemporaneous elasticities of sectoral output to productivity shocks, shocks to biophysical impacts, and impacts to meteorological variables. In addition, $\gamma_{7}$ is the elasticity of future carbon energy prices to extraction today, and $\gamma_{11}$ and $\gamma_{12}$ denote the elasticities of future meteorology to the current atmospheric GHG concentrations and the stock of future GHGs to current emissions-cum-energy use. Finally, we let $g_{t, t^{\prime}}^{P}=P_{t}^{E} / P_{t^{\prime}}^{E}$ and $g_{\ell^{\prime}, t, t^{\prime}}^{E}=Q_{\ell^{\prime}, t}^{E} / Q_{\ell^{\prime}, t^{\prime}}^{E}$ indicate the growth in the global price and region- $\ell^{\prime}$ quantity of energy use, and $\sigma_{\ell, \ell^{\prime}}^{Y}=Q_{\ell, t^{\prime}}^{Y} / Q_{\ell^{\prime}, t^{\prime}}^{Y}$ and 
$\sigma_{\ell, \ell^{\prime}}^{E}=Q_{\ell, t^{\prime}}^{E} / Q_{\ell^{\prime}, t^{\prime}}^{E}$ denote the relative shares of current world GDP and energy use.

Considerable manipulation yields:

$$
\begin{aligned}
& \frac{P_{t^{\prime}}^{E} Q_{\ell^{\prime}, t^{\prime}}^{E}}{Q_{\ell^{\prime}, t^{\prime}}^{Y}}=\left(1+\mathcal{S}_{\ell^{\prime}, t^{\prime}}\right)^{-1} \sum_{j=1}^{\mathcal{N}} \gamma_{1}\left[Q_{\ell^{\prime}}^{Y}, q_{j, \ell^{\prime}}^{Y}\right] \cdot \gamma_{5}\left[q_{j, \ell^{\prime}}^{Y}, q_{j, \ell^{\prime}}^{E}\right] \cdot \gamma_{6}\left[q_{j, \ell^{\prime}}^{E}, Q_{\ell^{\prime}}^{E}\right] \\
& +\left(1+\mathcal{S}_{\ell^{\prime}, t^{\prime}}\right)^{-1} \cdot \mathbb{E}_{t^{\prime}} \sum_{t=t^{\prime}+1}^{T} \beta^{\left(t-t^{\prime}\right)} \\
& \times \sum_{\ell=1}^{\mathcal{L}}\left\{\times \sum_{j=1}^{\mathcal{N}}\left\{\begin{array}{c}
\mathcal{D}_{\ell, t, t^{\prime}}\left[\sigma_{\ell, \ell^{\prime}}^{Y} g_{\ell, t, t^{\prime}}^{Y}, q_{j, \ell}^{Y}\right] \cdot \gamma_{8}\left[q_{j, \ell}^{Y}, \Lambda_{j, \ell}\right] \\
\times \sum_{i=1}^{J}\left[\times \sum_{m=1}^{\mathcal{M}}\left(\gamma_{10}\left[b_{j, \ell}^{i}, M_{\ell, t}^{m}\right] \cdot \sum_{s=t^{\prime}+1}^{t} \gamma_{11}^{i}, M_{j, \ell}^{i}\right]\right.
\end{array}\right]\right\}
\end{aligned}
$$

which is the sum of two effects: the contemporaneous elasticity of $\ell$ 's GDP to its energy

extraction —-mediated by sectoral structure, and the elasticity of discounted forgone future output in all regions to GHGs currently being emitted. Both factors are attenuated by the resource stock effect, given by

$$
\mathcal{S}_{\ell^{\prime}, t^{\prime}}=\mathbb{E}_{t^{\prime}} \sum_{t=t^{\prime}}^{T} \beta^{\left(t-t^{\prime}\right)} \gamma_{7}\left[P_{t}^{E}, Q_{\ell^{\prime}, t^{\prime}}^{E}\right] \cdot\left(\sum_{\ell=1}^{\mathcal{L}} \mathcal{D}_{\ell, t, t^{\prime}} \sigma_{\ell, \ell^{\prime}}^{E} g_{\ell, t, t^{\prime}}^{P} g_{\ell, t, t^{\prime}}^{E}\right)
$$

Extraction is decreasing in $\mathcal{S}$, so with a positive numerator in ( $\left.3^{\prime}\right)$, more rapid future increases in carbon-energy extraction and energy prices, and a more elastic response of future energy prices to current extraction will tend to lower the current value of energy use, all else equal. ${ }^{10}$

NRC (2010) emphasized that the challenge in computing the SCC arises from the terms in curly braces in (3). Interpreting this in light of eq. (3’), carbon cycle science is sufficiently advanced to enable us to establish values for $\gamma_{12}$ with a fair degree of confidence. Regarding $\gamma_{11}$,

\footnotetext{
${ }^{10}$ Interestingly, while a long-run carbon energy extraction cost function along the lines of (1d) were incorporated into early versions of RICE (Nordhaus and Boyer, 2000: eq. (2.12)) this feature is absent from recent variants of the model (Nordhaus, 2008; 2010), in all likelihood because the particular parameterization of extraction cost was inelastic over a broad range ( $\gamma_{7} \rightarrow 0$ even for large $t$, cf Nordhaus and Boyer, 2000: Fig. 3.4).
} 
climate models' ability to simulate the responses of future temperatures and sea levels at regional scales has substantially improved, though precipitation and ice/snow cover still pose challenges (Bader et al., 2008). But the bigger gap in our understanding stems from the lack comprehensive accounts in the form of Table 1 that track the responses of biophysical impacts to meteorology $\left(\gamma_{10}\right)$ and of economic productivity to these endpoints $\left(\gamma_{9}\right)$, especially the variation of the relevant elasticities across regions, sectors and impact categories. An additional layer of complexity is the moderating effects of adaptation on the elasticities themselves, which we now discuss.

Looking first at Type I adaptation, the primary feedback of market signals on extraction decisions only manifests itself after a long lag, when climate damages reduce energy’s output elasticity, $\gamma_{5}$. This delay cuts to the heart of the climate problem: because private actors do not perceive the negative second term in (3'), they face no incentive to slow fossil fuel use and emissions until climate change begins to exert significant adverse impacts on energy's economic productivity. An intertemporally optimizing social planner will impose a pigovian tax $(\tau)$ that internalizes the marginal cost of climate damages as a corrective measure. The definition of the SCC in (3) implies that the second term in (3') is a simple transformation of the optimal tax:

$$
-\left(1+\mathcal{S}_{t^{\prime}}\right)^{-1}\left(Q_{\ell^{\prime}, t^{\prime}}^{E} / Q_{\ell^{\prime}, t^{\prime}}^{Y}\right) \tau_{t^{\prime}} .
$$

Although the tax is globally uniform (reflecting the damage from a well-mixed pollutant) its attenuating effects on regions' extraction paths vary with their energy and emission intensity.

The fact that $\tau$ encompasses all of the climatic effects of interest emphasizes that actions to mitigate and adapt to climate change should not be thought of as separable. On the contrary they are inextricably linked, through adaptation's influence on the future trajectory of marginal damages and the potential for the latter to foreclose GHG mitigation options. The second effect 
is not widely appreciated. Imposition of $\tau$ disincentivizes fossil fuel production, raises the return to capital for zero-emission energy supply sectors such as nuclear and hydropower, and induces the latter to expand capacity and output. But these sectors may experience offsetting declines in the marginal product of capital due to the impacts of precipitation changes on cooling water supplies or stream flow. The upshot of these effects is higher marginal costs of GHG abatement and climate damage, and larger equilibrium values of $\tau$. Conversely, the countervailing influence of adaptation will tend to lower the time-path of the tax, but the further into the future impacts begin to bite, and the less elastic the respnse of damages to adaptation, the smaller this effect is likely to be.

\section{Reactive adaptation}

Faced with climate damage losses that are imminent, an optimizing decision maker will choose to allocate investment among a vector of reactive adaptation options, $\boldsymbol{\rho}$, until the marginal value of avoided contemporaneous damages (reversal of the decline in sectoral productivity in (1m) that reduces forgone output in (1c)) equals the marginal opportunity cost of expenditure (the associated reduction in aggregate consumption in (1f)). The optimal level of reactive investment is given by $\partial \mathcal{W} / \partial \rho_{j^{\prime}, \ell^{\prime}, t^{\prime}}^{i}=0$, which yields:

$$
\frac{\partial \Phi_{\ell^{\prime}}}{\partial q_{j^{\prime}, \ell^{\prime}, \ell^{\prime}}^{Y}} \cdot \frac{\partial \psi_{j^{\prime}, \ell^{\prime}, \ell^{\prime}}}{\partial \Lambda_{j^{\prime}, \ell^{\prime}, \ell^{\prime}}} \cdot\left(\frac{\partial \lambda_{j^{\prime}, \ell^{\prime}}}{\partial \rho_{j^{\prime}, \ell^{\prime}, t^{\prime}}^{i}}+\frac{\partial \lambda_{j^{\prime}, \ell^{\prime}}}{\partial b_{j^{\prime}, \ell^{\prime}, \ell^{\prime}}^{i}} \cdot \frac{\partial \zeta_{j^{\prime}, \ell^{\prime}}^{i}}{\partial \rho_{j^{\prime}, \ell^{\prime}, \ell^{\prime}}^{i}}\right)=1
$$

The first and second terms in brackets are the avoided marginal productivity losses due to adaptive and defensive components of expenditure, respectively. From Table 2, $\gamma_{13}$ and $\gamma_{14}$

denote the contemporaneous elasticities of the climate-induced productivity shock and the $i^{\text {th }}$ underlying biophysical impact endpoint to reactive investment. The optimal intensity of reactive adapation in each period is then: 


$$
\begin{aligned}
& \frac{\rho_{j^{\prime}, \ell^{\prime}}^{i}}{Q_{\ell^{\prime}}^{Y}}=\gamma_{1}\left[Q_{\ell^{\prime}}^{Y}, q_{j^{\prime}, \ell^{\prime}}^{Y}\right] \cdot \gamma_{8}\left[q_{j^{\prime}, \ell^{\prime}}^{Y}, \Lambda_{j^{\prime}, \ell^{\prime}}\right]
\end{aligned}
$$

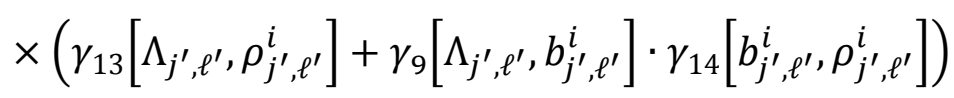

The intuitive result is that regions have incentives to spend a larger share of output on reactive adaptation the more elastic the contemporaneous response of GDP to the output of climaticallyexposed sectors, the more elastic sectors' outputs are to climate shocks, and the more elastic the shock is to the moderating effect of adaptation, through both defensive and adaptive channels. The strengths of these channels $\left(\gamma_{13}\right.$ and $\left.\gamma_{14}\right)$ are a key unknown, especially given our incomplete understanding of the difficulty of adaptation and how it might vary across regions and economic sectors.

\section{Proactive adaptation}

Building on Bosello et al (2010a,b), our IAM’s intertemporal structure captures the balance between discounted marginal future reductions in damages from proactive measures and the current marginal opportunity cost of the corresponding expenditure in (1f), and how it determines the incentive for the accumulation of stock adaptation capacity in (1i) prior to the onset of damages. An optimizing regional social planner will choose to allocate investment among a vector of proactive adaptation options, $\boldsymbol{\pi}$, until the present value of future savings from avoided damage equals the current opportunity cost of investment, which is given by the firstorder condition $\partial \mathcal{W} / \partial \pi_{j^{\prime}, \ell^{\prime}, t^{\prime}}^{i}=0$ : 


$$
\begin{aligned}
\mathbb{E}_{t^{\prime}} \sum_{t=t^{\prime}+1}^{T} \beta^{t}(1 & \left.-\vartheta^{i}\right)^{\left(t-t^{\prime}-1\right)} \frac{\partial U_{\ell^{\prime}}}{\partial Q_{\ell^{\prime}, t}^{C}} \cdot \frac{\partial \Phi_{\ell^{\prime}}}{\partial q_{j^{\prime}, \ell^{\prime}, t}^{Y}} \cdot \frac{\partial \psi_{j^{\prime}, \ell^{\prime}, t}}{\partial \Lambda_{j^{\prime}, \ell^{\prime}, t^{\prime}}} \\
& \times\left(\frac{\partial \lambda_{j^{\prime}, \ell^{\prime}}}{\partial a_{j^{\prime}, \ell^{\prime}, t}^{i}}+\frac{\partial \lambda_{j^{\prime}, \ell^{\prime}}}{\partial b_{j^{\prime}, \ell^{\prime}, t}^{i}} \cdot \frac{\partial \zeta_{j^{\prime}, \ell^{\prime}}^{i}}{\partial a_{j^{\prime}, \ell^{\prime}, t}^{i}}\right)=\beta^{t^{\prime}} \frac{\partial U_{\ell^{\prime}}}{\partial Q_{\ell^{\prime}, t^{\prime}}^{C}}
\end{aligned}
$$

Similar to eq. (4'), $\gamma_{15}-\gamma_{17}$ denote the elasticity of future adaptation capital to current proactive investment, and the elasticities of the climate shock and the $i^{\text {th }}$ biophysical endpoint with respect to the stock of proactive adaptation capacity. Then the optimal intensity of anticipatory adapation investment is: ${ }^{11}$

$$
\begin{gathered}
\frac{\pi_{j^{\prime}, \ell^{\prime}, t^{\prime}}^{i}}{Q_{\ell^{\prime}, t^{\prime}}^{Y}}=\mathbb{E}_{t^{\prime}} \sum_{t=t^{\prime}+1}^{T} \beta^{\left(t-t^{\prime}\right)} \mathcal{D}_{\ell^{\prime}, t} g_{\ell^{\prime}, \ell^{\prime}, t}^{Y} \cdot \gamma_{1}\left[Q_{\ell^{\prime}, t}^{Y}, q_{j^{\prime}, \ell^{\prime}, t}^{Y}\right] \cdot \gamma_{8}\left[q_{j^{\prime}, \ell^{\prime}, t}^{Y}, \Lambda_{j^{\prime}, \ell^{\prime}, t}\right] \\
\times \gamma_{15}\left[a_{j^{\prime}, \ell^{\prime}, t}^{i}, \pi_{j^{\prime}, \ell^{\prime}, t^{\prime}}^{i}\right] \cdot\left(\gamma_{16}\left[\Lambda_{j^{\prime}, \ell^{\prime}, t}, a_{j^{\prime}, \ell^{\prime}, t}^{i}\right]+\gamma_{9}\left[\Lambda_{j^{\prime}, \ell^{\prime}, t}, b_{j^{\prime}, \ell^{\prime}, t}^{i}\right] \cdot \gamma_{17}\left[b_{j^{\prime}, \ell^{\prime}, t^{\prime}}^{i}, a_{j^{\prime}, \ell^{\prime}, t}^{i}\right]\right)
\end{gathered}
$$

As above, current proactive adaptation generates a larger stream of future benefits the more elastic the response of future GDP to the output of climatically-exposed sectors, the more elastic sectoral output is to climate shocks, and the more elastic the shock is to the moderating effect of adaptation, through both defensive and adaptive channels. Additional intertemporal considerations are that benefits increase with the growth of future output, the durability of nearterm investments (in the sense of allowing future adaptation capacity to respond more elastically), and the rate of time preference is lower, reducing the effect of discounting. The key unknowns are the elasticities of endpoints and the productivity shocks to the stock of adaptation capacity, $\gamma_{16}$ and $\gamma_{17}$.

\footnotetext{
${ }^{11}$ Similar to footnote $5,\left(1-\vartheta^{i}\right)^{\left(t-t^{\prime}-1\right)}=\gamma_{6}\left[a_{j^{\prime}, \ell^{\prime}}^{i}, \pi_{j^{\prime}, \ell^{\prime}}^{i}\right] a_{j^{\prime}, \ell^{\prime}}^{i} / \pi_{j^{\prime}, \ell^{\prime}}^{i}$
} 


\section{Discussion}

We draw particular attention to the fact that eqs. (2'), (3’), (4’) and (5') are not independent. Indeed, this system of equations constitutes a fixed point problem in which one control variable affects the values of key elasticities that determines the other controls. An unsatisfying aspect of our analysis is of course our inability to calibrate the values of the constituent variables, or evaluate the impact of optimal mitigation and adaptation strategies on sectoral growth and regional welfare. ${ }^{12}$ Indeed, the latter requires the full specification, parameterization and solution of our IAM, but the inability of current empirical research to support parameterization of the elasticities in Table 2 proves to be an insurmountable roadblock. As we elaborate in a subsequent paper, engineering and econometric estimates of impacts’ consequences cannot be easily translated into a form suitable for our IAM because of inadequate regional and sectoral coverage, and incomplete understanding of many potentially consequential biophysical endpoint channels.

This situation can only be remedied by systematic enumeration of the sets of potentially economically relevant meteorological and endpoint variables ( $m$ and $i$ ) and their linkages to economic sectors, as well as research to characterize and measure the efficacy of related proactive and reactive adaptation responses. The elasticities $\gamma_{8}-\gamma_{10}$ and $\gamma_{13}-\gamma_{17}$ are particular targets for investigation. However, given the diversity of the channels through which meteorology might end up impacting the productivity of various sectors, it is admittedly simpler to aggregate together different biophysical impacts and consider aggregated region-by-sector impacts and adaptation responses. The issue is what if any important elements might be lost in this sort of aggregation, and what biases might thereby be introduced into IAMs' representations

\footnotetext{
${ }^{12}$ It is possible to substitute the optimal values of our controls into the capacity constraint (2f) to solve for the level of period- $t^{\prime}$ consumption, and, with (2a), welfare. However, even the single-period algebraic result is complicated enough to defy easy interpretation.
} 
of climate shocks. We feel it is important to ask this question, but currently lack the means to arrive at an answer.

Notwithstanding this, insights can still be drawn about how near-term mitigation and adaptation actions can be induced by expectations of impacts. The traditional procedure relies on two key simplifying assumptions (cf Golosov et al, 2011; Krusell and Hassler, 2012). The first is to hold constant the expected values of the elasticities in Table 2, treating them as parameters (indicated by a bar). The second is to treat the probability density functions (PDFs) of the elasticities and the growth rates of economic variables as independent of one another. Then, assuming current climate damages are zero, (3') and (5') imply that the initial values of the optimal GHG tax and intensity of proactive adaptation at $t^{\prime}=0$ are:

$$
\begin{gathered}
\tau_{0} \approx \sum_{t=1}^{T} \beta^{t} \sum_{\ell=1}^{\mathcal{L}}\left\{\frac{Q_{\ell, 0}^{Y}}{Q_{\ell, 0}^{E}} \overline{\mathcal{D}}_{\ell, t} \bar{g}_{\ell, t}^{Y} \cdot \sum_{j=1}^{\mathcal{N}}\left\{\bar{\gamma}_{1} \bar{\gamma}_{8} \cdot \sum_{i=1}^{\mathcal{J}}\left[\bar{\gamma}_{9} \cdot \sum_{m=1}^{\mathcal{M}}\left(\bar{\gamma}_{10} \cdot \sum_{s=1}^{t} \bar{\gamma}_{11}(t, s) \cdot \bar{\gamma}_{12}(s, 0)\right)\right]\right\}\right\}, \\
\pi_{j^{\prime}, \ell^{\prime}, 0}^{i} \approx Q_{\ell^{\prime}, 0}^{Y} \cdot \sum_{t=1}^{T} \beta^{t} \overline{\mathcal{D}}_{\ell^{\prime}, t} \bar{g}_{\ell^{\prime}, t}^{Y} \cdot \bar{\gamma}_{1} \bar{\gamma}_{8} \bar{\gamma}_{15} \cdot\left(\bar{\gamma}_{16}+\bar{\gamma}_{9} \bar{\gamma}_{17}\right) .
\end{gathered}
$$

While these expressions provide a rough-and-ready means to characterize optimal nearterm policies (subject to judgments of, or hard numbers on, elasticities and growth forecasts), they suffer from unknown bias relative to their true values. The problem is the assumption of independence. It enables the analyst to sidestep the complication that the expectation operators in (3') and (5') are denominated over a nested set of inner products of random variables, but at the cost of ignoring covariances among and between elasticities and growth rates whose values might be significant. ${ }^{13}$

The magnitude of the latter second-order impact is very much a measure of our ignorance

\footnotetext{
${ }^{13}$ Recall that for non-independent random variables $A$ and $B, \mathbb{E}[A B]=\mathbb{E}[A] \mathbb{E}[B]+\operatorname{Cov}[A B]$.
} 
about climate damages and adaptation to them. Not only is this effect ripe for theoretical and empirical investigation, it indicates a need for caution when using point estimates from empirical climate impact studies as parameters in IAMs. Given the considerable scope of uncertainties, a more robust approach would be to use a mix of empirical and simulation evidence, judgment and assumptions to construct PDFs of the relevant elasticities and growth rates, sample intensively from these distrbutions assuming a variety of correlation structures, use the model parameter sets to perform Monte Carlo simulation of the control variables, and, finally, from the output PDFs of $\tau_{0}$ and $\pi_{j^{\prime}, \ell^{\prime}, 0}^{i}$, recover the expected values.

We close by briefly touching on technological change. Despite much progress characterizing and modeling innovation in GHG abatement or fossil fuel substitute technologies, the few IAMs that explicitly represent adaptation do not explicitly consider improvements in related technology options, to say nothing of those induced by increased demand for proactive and/or defensive measures. This too arises from our limited understanding of the processes by which innovation in this area proceeds, and the consequent paucity of relevant empirical estimates on which to base model parameterizations. Structurally, adaptation-related autonomous productivity improvements or learning-by-doing can be easily accommodated in our IAM by specifiying time- or investment-dependent increases in the magnitudes of the elasticities $\gamma_{13}, \gamma_{14}$, $\gamma_{16}$ and $\gamma_{17}$. The caveat is the lack of evidence for such structural and parametric choices, and the research that will be required to establish the necessary basis. Since future climatic changes — and societal responses to them—are projected to be outside the range of historical experience, we may need to draw insights from investigation of analogous reactive or adaptive innovations (e.g., those induced by natural disasters). 


\section{Summary}

This paper has developed a framework for modeling climate impacts and adaptation. We first identified five characteristics of an ideal IAM: regional and sectoral detail for impacts and adaptation strategies; distinct representation of the three types of adaptation-via market adjustments, protective/defensive expenditures, and adaptive/coping expenditures; intertemporal decision making under uncertainty; induced innovation in adaptation-related technologies; and connection with empirical work on impacts and adaptation. From these desiderata we developed a conceptual framework that we elaborated into an impacts- and adaptation-centric IAM. Using the model's optimality conditions, we then identified the types of functional relationships that IAMs need to incorporate in order to more realistically represent adaptation-related decisions, and the ways in which the inclusion of these elements might affect IAMs’ results.

Throughout our discussion, a recurring theme was the urgent need for empirical estimates upon which model parameterizations. This issue is the focus of a subsequent paper, in which we review available empirical estimates and assess their appropriateness for incorporation within IAMs, and examine the empirical content of methods currently used to parameterize IAMs. 


\section{References}

1. Anthoff, D. and S.K. Rose and R.S.J. Tol and S. Waldhoff (2011). Regional and Sectoral Estimates of the Social Cost of Carbon: An Application of FUND, Economic and Social Research Institute Working Paper No. WP375.

2. Bosello, F., C. Carraro and E. De Cian (2010a). Climate Policy and the Optimal Balance between Mitigation, Adaptation and Unavoided Damage, Climate Change Economics 1: 7192.

3. Bosello, F., C. Carraro and E. De Cian (2010b). An Analysis of Adaptation as a Response to Climate Change, in B. Lomborg (ed.), Smart Solutions to Climate Change, Cambridge: Cambridge University Press, 222-276.

4. Fishback, P.V., W. Troesken, T. Kollmann, M. Haines, P.W. Rhode and M. Thomasson (2011). Information and the Impact of Climate and Weather on Mortality Rates During the Great Depression, in G.D. Libecap and R.H. Steckel (eds.), The Economics of Climate Change: Adaptations Past and Present, Chicago: University of Chicago Press, 131-167.

5. Hansen, Z.K., G.D. Libecap and S.E. Lowe (2011). Climate Variability and Water Infrastructure: Historical Experience in the Western United States, in G.D. Libecap and R.H. Steckel (eds.), The Economics of Climate Change: Adaptations Past and Present, Chicago: University of Chicago Press, 253-280.

6. Hassler, J., and P. Krusell (2012). Economics and Climate Change: Integrated Assessment in a Multi-Region World, National Bureau of Economic Research Working Paper No. 17757.

7. Golosov, M., J. Hassler, P. Krusell and A. Tsyvinski (2011). Optimal Taxes on Fossil Fuel in General Equilibrium, National Bureau of Economic Research Working Paper No. 17348. 
8. Graff Zivin, J and M.J. Neidell (2010). Temperature and the Allocation of Time:

Implications for Climate Change, National Bureau of Economic Research Working Paper No. 15717.

9. Landon-Lane, J., H. Rockoff and R.H. Steckel (2011). Droughts, Floods and Financial Distress in the United States, in G.D. Libecap and R.H. Steckel (eds.), The Economics of Climate Change: Adaptations Past and Present, Chicago: University of Chicago Press, 73-98.

10. Libecap, G.D. and R.H. Steckel (eds.) (2011). The Economics of Climate Change: Adaptations Past and Present, University of Chicago Press, Chicago.

11. National Research Council (NRC) (2010). Hidden Costs of Energy: Unpriced Consequences of Energy Production and Use, National Academies Press, Washington, DC.

12. Nordhaus, W.D. (2008). A Question of Balance: Weighing the Options on Global Warming Policies, Yale University Press.

13. Nordhaus, W.D. (2010). Economic aspects of global warming in a post-Copenhagen environment, Proceedings of the National Academy of Sciences 107: 11721-11726.

14. Nordhaus, W.D., and J. Boyer (2001). Warming the World: Economic Models of Global Warming, MIT Press.

15. Tol, R.S.J. (1995). The damage costs of climate change: toward more comprehensive calculations, Environmental and Resource Economics 5: 353-374. 
Figure 1. Economic Damages from Climate Impacts: A Bottom-Up Framework

(i) Change in Global Atmospheric GHG Concentrations

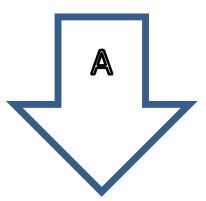

(ii) Changes in Climate Variables (by Region)

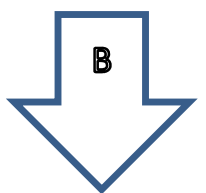

(iii) Response of Physical Impact Endpoints to Climate Variables (by Region)

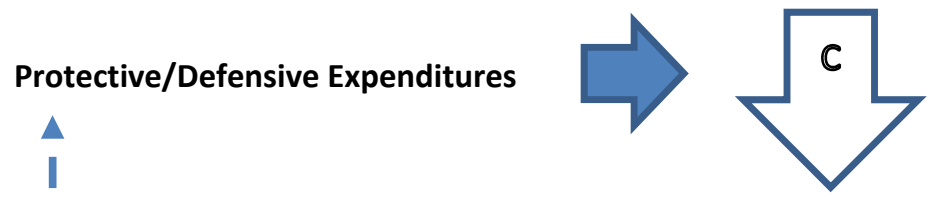

(iv) Response of Sectoral Productivities to Physical Impact Endpoints (by Region and Sector)

I

| Adaptive/Coping Expenditures

I

(
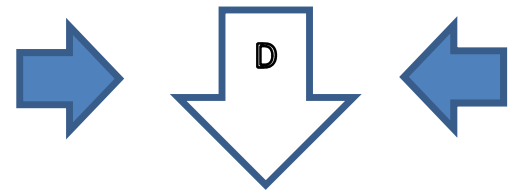

General Equilibrium Effects

(v) Economic Losses (by Region and Sector)

4

L

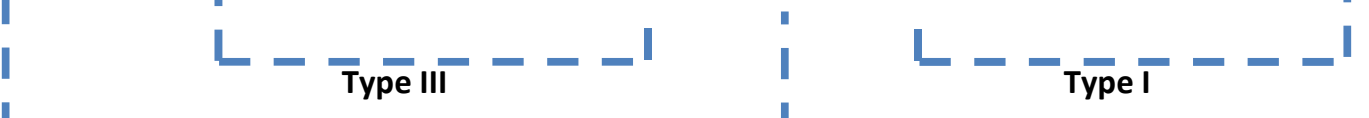

L $----\frac{-}{\text { Type II }}-----J$ 
Figure 2. A Canonical IAM Incorporating Climate Impacts and Adaptation

A. Nomenclature

\begin{tabular}{cl}
\hline Set indexes: & \\
$t=\{0, \ldots, \mathcal{T}\}$ & Time periods \\
$\ell=\{1, \ldots, \mathcal{L}\}$ & World regions \\
$j=\{1, \ldots, \mathcal{N}\}$ & Industry sectors \\
$m=\{1, \ldots, \mathcal{M}\}$ & Meteorological characteristics \\
$i=\{1, \ldots, \mathcal{J}\}$ & Climate impact endpoints \\
Control variables: & \\
$q_{j, \ell, t}^{E}$ & Sectoral energy input \\
$q_{j, \ell, t}^{K}$ & Sectoral capital input \\
$Q_{\ell, t}^{C}$ & Aggregate consumption \\
$X_{\ell, t}^{K}$ & Aggregate jelly capital investment \\
$\rho_{j, \ell, t}^{i}$ & Region-, sector- and impact-specific reactive adaptation expenditure \\
$\pi_{j, \ell, t}^{i}$ & Region-, sector- and impact-specific proactive adaptation investment \\
Economic state variables: \\
$\mathcal{W}$ & Welfare (model objective) \\
$q_{j, \ell, t}^{Y}$ & Net sectoral product \\
$Q_{\ell, t}^{Y}$ & Aggregate net regional product \\
$Q_{\ell, t}^{E}$ & aggregate regional energy use \\
$P_{t}^{E}$ & Global marginal energy resource extraction cost \\
$Q_{\ell, t}^{K}$ & Stock of aggregate jelly capital \\
$a_{j, \ell, t}^{i}$ & Stock of region-, sector- and impact-specific proactive adaptation capital \\
Environmental state variables: \\
$G_{t}$ & Global stock of atmospheric GHGs \\
$M_{\ell, t}^{m}$ & Region-specific climate variables \\
$b_{j, \ell, t}^{i}$ & Region-, sector-, and impact-specific endpoint indexes \\
$\Lambda_{j, \ell, t}$ & Region- and sector-specific damage induced productivity losses \\
Functional relationships: & Global intertemporal welfare \\
$\Xi$ & Regional intratemporal utility \\
$U_{\ell}$ & Regional aggregate production functions \\
$\Phi_{\ell}$ & Sectoral production functions \\
$\psi_{j, \ell}$ & Global energy supply function \\
$\Theta$ & Regional climate response functions \\
$\mathcal{E}$ & Regional and sectoral climate impacts functions \\
$Y_{\ell}^{m}$ & \\
$\zeta_{j, \ell}^{i}$ & \\
$\lambda_{j, \ell}$ & \\
\hline &
\end{tabular}


Objective:

$$
\max _{x_{\ell, t}^{K}, Q_{\ell, t}^{E}, \rho_{j, \ell, t}^{i}, \pi_{j, \ell, t}^{i}, q_{j, \ell, t}^{K}, q_{j, \ell, t}^{E}} \mathcal{W}=\mathbb{E}_{0} \sum_{t=0}^{T} \beta^{t} \Xi\left[U_{1}\left[Q_{1, t}^{C}\right], \ldots, U_{\mathcal{L}}\left[Q_{\mathcal{L}, t}^{C}\right]\right]
$$

Aggregate net regional product:

$$
Q_{\ell, t}^{Y}=\Phi_{\ell}\left[q_{1, \ell, t}^{Y}, \ldots, q_{\mathcal{N}, \ell, t}^{Y}\right]
$$

Sectoral net regional product:

$$
q_{j, \ell, t}^{Y}=\psi_{j, \ell}\left[q_{j, \ell, t}^{E}, q_{j, \ell, t}^{K} ; \Lambda_{j, \ell, t}\right]
$$

Intra-regional and intra-temporal market clearance for energy:

$$
\sum_{j=1}^{\mathcal{N}} q_{j, \ell, t}^{E}=Q_{\ell, t}^{E}
$$

Intra-regional and intra-temporal market clearance for jelly capital:

$$
\sum_{j=1}^{\mathcal{N}} q_{j, \ell, t}^{K}=Q_{\ell, t}^{K}
$$

Aggregate regional absorption constraint:

$$
Q_{\ell, t}^{C}=Q_{\ell, t}^{Y}-X_{\ell, t}^{K}-P_{t}^{E} Q_{\ell, t}^{E}-\sum_{i=1}^{j} \sum_{j=1}^{\mathcal{N}}\left(\rho_{j, \ell, t}^{i}+\pi_{j, \ell, t}^{i}\right)
$$

Global energy trade and marginal resource extraction cost:

$$
P_{t}^{E}=\Theta\left[\sum_{\ell=1}^{\mathcal{L}} \sum_{s=0}^{t} Q_{\ell, s}^{E}\right]
$$

Regional jelly capital accumulation:

$$
Q_{\ell, t+1}^{K}=X_{\ell, t}^{K}+\left(1-\vartheta^{K}\right) Q_{\ell, t}^{K}
$$

Accumulation of impact-, sector- and region- specific adaptation capital:

$$
a_{j, \ell, t+1}^{i}=\pi_{j, \ell, t}^{i}+\left(1-\vartheta^{i}\right) a_{j, \ell, t}^{i}
$$

\section{Climate Sub-Model}

Global atmospheric GHG accumulation:

$$
G_{t+1}=\varepsilon_{t}\left[\sum_{\ell} Q_{\ell, t}^{E}, G_{t}\right]
$$

Regional meteorological effects of global atmospheric GHG concentration:

$$
M_{\ell, t}^{m}=\Upsilon_{\ell, t}^{m}\left[G_{t}, G_{t-1}, G_{t-2}, \ldots\right]
$$

\section{Impacts Sub-Model}

Biophysical climate impacts by type, sector and region:

$$
b_{j, \ell, t}^{i}=\zeta_{j, \ell}^{i}\left[M_{\ell, t}^{1}, \ldots, M_{\ell, t}^{\mathcal{M}} ; \rho_{j, \ell, t}^{i} ; a_{j, \ell, t}^{i}\right]
$$

Climate-induced productivity shocks:

$$
\Lambda_{j, \ell, t}=\lambda_{j, \ell}\left[b_{j, \ell, t}^{1}, \ldots, b_{j, \ell, t}^{\jmath} ; \rho_{j, \ell, t}^{1}, \ldots, \rho_{j, \ell, t}^{\jmath} ; a_{j, \ell, t}^{1}, \ldots, a_{j, \ell, t}^{\jmath}\right]
$$


Table 1. Key Responses in Modeling Climate Impacts and Adaptation

A. Regional Responses of Impact Endpoints to Climate Variables

\begin{tabular}{|c|c|c|c|c|c|}
\hline \multirow[t]{2}{*}{ Region } & \multirow{2}{*}{$\begin{array}{c}\text { Impact } \\
\text { Endpoint }\end{array}$} & \multirow{2}{*}{$\begin{array}{c}\text { Climate } \\
\text { Variable }\end{array}$} & \multirow{2}{*}{$\begin{array}{c}\text { Base } \\
\text { Response }\end{array}$} & \multicolumn{2}{|c|}{ Effect of Adaptation } \\
\hline & & & & Reactive & Proactive \\
\hline \multirow{9}{*}{ Region 1} & \multirow{4}{*}{ Endpoint 1} & Temperature & & & \\
\hline & & Precipitation & & & \\
\hline & & Sea Level & & & \\
\hline & & $\ldots$ & & & \\
\hline & $\ldots$ & & & & \\
\hline & \multirow{4}{*}{ Endpoint $i$} & Temperature & & & \\
\hline & & Precipitation & & & \\
\hline & & Sea Level & & & \\
\hline & & $\ldots$ & & & \\
\hline$\ldots$ & & & & & \\
\hline \multirow{9}{*}{ Region $\mathcal{L}$} & \multirow{4}{*}{ Endpoint 1} & Temperature & & & \\
\hline & & Precipitation & & & \\
\hline & & Sea Level & & & \\
\hline & & $\ldots$ & & & \\
\hline & $\ldots$ & & & & \\
\hline & \multirow{4}{*}{ Endpoint $\mathcal{J}$} & Temperature & & & \\
\hline & & Precipitation & & & \\
\hline & & Sea Level & & & \\
\hline & & & & & \\
\hline
\end{tabular}

B. Sectoral Responses of Productivities to Impact Endpoints

\begin{tabular}{|c|c|c|c|c|c|}
\hline \multirow[t]{2}{*}{ Region } & \multirow[t]{2}{*}{ Sector } & \multirow{2}{*}{$\begin{array}{l}\text { Impact } \\
\text { Endpoint }\end{array}$} & \multirow{2}{*}{$\begin{array}{c}\text { Base } \\
\text { Response }\end{array}$} & \multicolumn{2}{|c|}{ Effect of Adaptation } \\
\hline & & & & Reactive & Proactive \\
\hline \multirow{7}{*}{ Region 1} & \multirow{3}{*}{ Sector 1} & Endpoint 1 & & & \\
\hline & & $\ldots$ & & & \\
\hline & & Endpoint $\mathcal{J}$ & & & \\
\hline & $\ldots$ & & & & \\
\hline & \multirow{3}{*}{ Sector $\mathcal{N}$} & Endpoint 1 & & & \\
\hline & & $\ldots$ & & & \\
\hline & & Endpoint $\mathcal{J}$ & & & \\
\hline$\ldots$ & & & & & \\
\hline \multirow{7}{*}{ Region $\mathcal{L}$} & \multirow{3}{*}{ Sector 1} & Endpoint 1 & & & \\
\hline & & $\ldots$ & & & \\
\hline & & Endpoint $\mathcal{J}$ & & & \\
\hline & $\ldots$ & & & & \\
\hline & \multirow{3}{*}{ Sector $\mathcal{N}$} & Endpoint 1 & & & \\
\hline & & $\ldots$ & & & \\
\hline & & Endpoint $\mathcal{J}$ & & & \\
\hline
\end{tabular}


Table 2. Key Elasticities in Modeling Climate Impacts and Adaptation

\begin{tabular}{|c|c|}
\hline$\gamma_{1}\left[Q_{\ell^{\prime}}^{Y}, q_{j^{\prime}, \ell^{\prime}}^{Y}\right]$ & Contemporaneous elasticity of region $\ell^{\prime \prime}$ s aggregate output to the output of sector $j^{\prime}$ \\
\hline$\gamma_{2}\left[q_{j^{\prime}, \ell^{\prime}}^{Y}, q_{j^{\prime}, \ell^{\prime}}^{K}\right]$ & Contemporaneous elasticity of output in sector $j^{\prime}$ and region $\ell^{\prime}$ to its use of capital \\
\hline$\gamma_{3}\left[q_{j^{\prime}, \ell^{\prime}}^{K}, Q_{\ell^{\prime}}^{K}\right]$ & Contemporaneous elasticity of sector $j^{\prime}$ use of capital to aggregate capital supply \\
\hline$\gamma_{4}\left[Q_{\ell, t}^{K}, Q_{\ell, t^{\prime}}^{K}\right]$ & Elasticity of period- $t$ aggregate capital to period- $t^{\prime}$ aggregate investment \\
\hline$\gamma_{5}\left[q_{j^{\prime}, \ell^{\prime}}^{Y}, q_{j^{\prime}, \ell^{\prime}}^{E}\right]$ & Contemporaneous elasticity of output to energy input in sector $j^{\prime}$ and region $\ell^{\prime}$ \\
\hline$\gamma_{6}\left[q_{j, \ell^{\prime}}^{E}, Q_{\ell^{\prime}}^{E}\right]$ & Contemporaneous elasticity of sector $j^{\prime}$ energy use to region $\ell^{\prime}$ carbon-energy extraction \\
\hline$\gamma_{7}\left[P_{t}^{E}, Q_{\ell^{\prime}, t^{\prime}}^{E}\right]$ & $\begin{array}{l}\text { Elasticity of the period-t global energy price with respect to carbon-energy extraction in } \\
\text { region } \ell^{\prime} \text { in period } t^{\prime}<t\end{array}$ \\
\hline$\gamma_{8}\left[q_{j^{\prime}, \ell^{\prime}}^{Y}, \Lambda_{j^{\prime}, \ell^{\prime}}\right]$ & $\begin{array}{l}\text { Contemporaneous elasticity of sector } j^{\prime \prime} \text { s output with respect to the climate-induced } \\
\text { productivity shock in region } \ell^{\prime}\end{array}$ \\
\hline$\gamma_{9}\left[\Lambda_{j^{\prime}, \ell^{\prime}}, b_{j^{\prime}, \ell^{\prime}}^{i}\right]$ & $\begin{array}{l}\text { Contemporaneous elasticity of the productivity shock to sector } j^{\prime} \text { in region } \ell^{\prime} \text { with respect } \\
\text { to the } i^{\text {th }} \text { biophysical endpoint }\end{array}$ \\
\hline$\gamma_{10}\left[b_{j, \ell}^{i}, M_{\ell, t}^{m}\right]$ & $\begin{array}{l}\text { Contemporaneous elasticity of the } i^{\text {th }} \text { biophysical endpoint impacting sector } j^{\prime} \text { in region } \\
\ell^{\prime} \text { with respect to climate variable } m\end{array}$ \\
\hline$\gamma_{11}\left[M_{\ell, t}^{m}, G_{s}\right]$ & Elasticity of climate variable $m$ in period $t$ to global atmospheric GHG stock in period $s<t$ \\
\hline$\gamma_{12}\left[G_{s}, Q_{\ell^{\prime}, t^{\prime}}^{E}\right]$ & $\begin{array}{l}\text { Elasticity of the period-s global atmospheric GHG stock to carbon-energy extraction in } \\
\text { period } t^{\prime}<s\end{array}$ \\
\hline$\gamma_{13}\left[\Lambda_{\left.j^{\prime}, \ell^{\prime}, \rho_{j^{\prime}, \ell^{\prime}}^{i}\right]}\right]$ & $\begin{array}{l}\text { Contemporaneous elasticity of the productivity shock to sector } j^{\prime} \text { in region } \ell^{\prime} \text { with respect } \\
\text { to reactive adapatation targeted at the } i^{\text {th }} \text { biophysical endpoint }\end{array}$ \\
\hline$\gamma_{14}\left[b_{j^{\prime}, \ell^{\prime}}^{i}, \rho_{j^{\prime}, \ell^{\prime}}^{i}\right]$ & $\begin{array}{l}\text { Contemporaneous elasticity of the } i^{\text {th }} \text { biophysical endpoint impacting sector } j^{\prime} \text { in region } \ell^{\prime} \\
\text { with respect to reactive investment }\end{array}$ \\
\hline$\gamma_{15}\left[a_{j^{\prime}, \ell^{\prime}}^{i}, \pi_{j^{\prime}, \ell^{\prime}}^{i}\right]$ & $\begin{array}{l}\text { Elasticity of future adaptation capital in sector } j^{\prime} \text { and region } \ell^{\prime} \text { with respect to current } \\
\text { proactive adaptation to the } i^{\text {th }} \text { biophysical endpoint }\end{array}$ \\
\hline$\gamma_{16}\left[\Lambda_{j^{\prime}, \ell^{\prime}}, a_{j^{\prime}, \ell^{\prime}}^{i}\right]$ & $\begin{array}{l}\text { Contemporaneous elasticity of the productivity shock to sector } j^{\prime} \text { in region } \ell^{\prime} \text { with respect } \\
\text { to the stock of proactive adaptation to the } i^{\text {th }} \text { biophysical endpoint }\end{array}$ \\
\hline$\gamma_{17}\left[b_{j^{\prime}, \ell^{\prime}}^{i}, a_{j^{\prime}, \ell^{\prime}}^{i}\right]$ & $\begin{array}{l}\text { Contemporaneous elasticity of the } i^{\text {th }} \text { biophysical endpoint impacting sector } j^{\prime} \text { in region } \\
\ell^{\prime} \text { with respect to the stock of proactive adaptation capacity }\end{array}$ \\
\hline
\end{tabular}

